\title{
Some Properties of the Signless Laplacian and Normalized Laplacian Tensors of General Hypergraphs
}

\author{
Cunxiang Duan, Ligong Wang* and Xihe Li
}

\begin{abstract}
In this paper, we obtain some properties of signless Laplacian eigenvalues of general hypergraphs. We give the upper and the lower bound of edge connectivity of general hypergraphs in terms of average degree, minimum degree, the rank and the number of vertices, or analytic connectivity $\alpha(G)$, respectively. We also give the upper bound of analytic connectivity $\alpha(G)$ of general hypergraphs in terms of the degrees of vertices. Finally, we obtain the bounds of the smallest $H^{+}$-eigenvalue of the normalized Laplacian sub-tensors of general hypergraphs.
\end{abstract}

\section{Introduction}

A hypergraph $H$ is a pair $(V, E)$, where $E \subseteq P(V)$ and $P(V)$ stands for the power set of $V$. The elements of $V=V(H)$ are called vertices, and the elements of $E=E(H)$ are called edges. If each edge $e \in E$ contains precisely $m$ vertices, then the hypergraph $H$ is called $m$-uniform. If $H=(V, E)$ and $H^{\prime}=\left(V^{\prime}, E^{\prime}\right)$ are hypergraphs such that $V^{\prime} \subseteq V$ and $E^{\prime} \subseteq E$, then $H^{\prime}$ is called a subhypergraph of $H$. For a vertex $v \in V$, we denote by $E_{v}$ the set of edges containing $v$, that is, $E_{v}=\{e \in E \mid v \in e\}$. The cardinality $\left|E_{v}\right|$ of the set $E_{v}$ is defined as the degree $d_{v}=d_{H}(v)$ of the vertex $v$. Similarly, for a subset $S \subset[n]=\{1,2, \ldots, n\}$, we denoted by $E_{S}$ the set of edges $\{e \in E \mid S \cap e \neq \emptyset\}$. The rank of $H$, denoted by $\operatorname{rank}(H)$, is the maximum cardinality of the edges in the hypergraph $H$. A nearly $m$-uniform supertree is both connected and acyclic, in which each edge contains either $m-1$ or $m$ vertices.

Spectral graph theory has a long history behind its development [2, 3, 5]. It is natural to generalize spectral graph theory from graphs to hypergraphs. In 2005, Qi [18] and Lim [12] independently introduced the concept of the eigenvalues of tensors and the spectra of tensors. In 2008, Lim [13] proposed the study of the spectra of hypergraphs by using the spectra of tensors. Recently, there are lots of papers concerning spectral hypergraph theory $4,6,8,10,17,19,21,24$. Li et al. 9 gave some operations such as

Received December 23, 2018; Accepted June 23, 2019.

Communicated by Xuding Zhu.

2010 Mathematics Subject Classification. 05C50, 15A18, 05C40.

Key words and phrases. signless Laplacian tensor, normalized Laplacian tensor, general hypergraph, edge connectivity, $H^{+}$-eigenvalue.

*Corresponding author. 
moving edges, edge-releasing and total grafting on hypergraphs, and determined the first two spectral radius of $m$-uniform supertrees with $n$ vertices. Lu and Man [16] generalized Smith's theorem to $m$-uniform hypergraphs, and classified all connected $m$-uniform hypergraphs with spectral radius at most $\rho_{m}=\sqrt[m]{4}$ which is the smallest limit point of the spectral radii of connected $m$-uniform hypergraphs. Xiao et al. 23 investigated the spectral radius of the $k$-uniform hypergraph when the uniform hypergraph is perturbed by 2 -switch operation, and determined the maximum spectral radius of $k$-uniform supertrees with given a degree sequence. Xiao et al. 22] determined the first two largest spectral radii of uniform supertrees with given diameter. Liu et al. 15 investigated the bounds of the principal ratio $\gamma(H)=x_{\max } / x_{\min }$ and gave an estimate of the gap of spectral radii between $H$ and its proper sub-hypergraph $H^{\prime}$. Many scholars also started to investigate the signless Laplacian spectral radius of $m$-uniform hypergraphs $7,14,25$.

In 2003, Qi [20] investigated the $\mathrm{H}^{+}$-eigenvalues of Laplacian tensors and signless Laplacian tensors. Banerjee et al. [1] gave some spectral properties of those hypermatrices of a general hypergraph, and found that these properties are similar for graphs and uniform hypergraphs. Zhang et al. 26 studied Perron-Frobenius theorem, the techniques of weighted incidence matrix and moving edges of general hypergraphs. And they determined the maximum spectral radius in two classes of nearly uniform supertrees. Inspired by the idea of Qi [20] and Banerjee et al. [1], we continue to study properties of the signless Laplacian and normalized Laplacian tensors of general hypergraphs.

In Section 2, some necessary notations and definitions are given. In Section 3, we mainly give some properties of signless Laplacian spectral of general hypergraphs, such as the bounds of $\mathrm{H}^{+}$-eigenvalues of the signless Laplacian tensors and the relationship of the signless Laplacian spectral radius of cartesian product of two hypergraphs and so on. In Section 4 , we mainly give the bounds of edge connectivity of general hypergraphs, the bound of analytic connectivity $\alpha(G)$ of general hypergraphs and the bounds of the smallest $H^{+}$-eigenvalue of the normalized Laplacian sub-tensors of general hypergraphs, respectively.

\section{Preliminaries}

A tensor $\mathcal{A}$ with order $m$ and dimension $n$ over the real field $\mathbb{R}$ is a multidimensional array

$$
\mathcal{A}=\left(a_{i_{1} i_{2} \ldots i_{m}}\right), \quad 1 \leq i_{1}, i_{2}, \ldots, i_{m} \leq n
$$

The tensor $\mathcal{A}$ is called symmetric if its entries are invariant under any permutation of their indices.

For a vector $x=\left(x_{1}, x_{2}, \ldots, x_{n}\right)^{T} \in \mathbb{R}^{n}, \mathcal{A} x^{m-1}$ is a vector in $\mathbb{R}^{n}$ with its $i$-th compo- 
nent defined as

$$
\left(\mathcal{A} x^{m-1}\right)_{i}=\sum_{i_{2}, \ldots, i_{m}=1}^{n} a_{i i_{2} \ldots i_{m}} x_{i_{2}} \cdots x_{i_{m}}, \quad \forall i \in[n] .
$$

Also, a tensor $\mathcal{A}$ of order $m$ and dimension $n$ uniquely determines an $m$ th degree homogeneous polynomial function $\mathcal{A} x^{m}$, which is

$$
x^{T}\left(\mathcal{A} x^{m-1}\right)=\sum_{i_{1}, i_{2}, \ldots, i_{m}=1}^{n} a_{i_{1} i_{2} \ldots i_{m}} x_{i_{1}} x_{i_{2}} \cdots x_{i_{m}} .
$$

Definition 2.1. 20 Let $\mathcal{A}$ be a nonzero tensor with order $m$ and dimension $n$. A pair $(\lambda, x) \in \mathbb{C} \times\left(\mathbb{C}^{n} \backslash\{0\}\right)$ is called eigenvalue and eigenvector (or simply an eigenpair) if they satisfy the following equation

$$
\mathcal{A} x^{m-1}=\lambda x^{[m-1]} \text {. }
$$

For $x^{[m-1]}$ is a vector with $i$-th entry $x_{i}^{m-1}$. We call $(\lambda, x)$ an $H$-eigenpair (i.e., $\lambda$ and $x$ are called $H$-eigenvalue and $H$-eigenvector, respectively) if they are both real. An $H$ eigenvalue $\lambda$ is called an $H^{+}\left(H^{++}\right)$-eigenvalue if the corresponding eigenvector $x \in \mathbb{R}_{+}^{n}$ $\left(\mathbb{R}_{++}^{n}\right)$.

Definition 2.2. [1] Let $H=(V, E)$ be a hypergraph with $V=\left\{v_{1}, v_{2}, \ldots, v_{n}\right\}$ and $E=\left\{e_{1}, e_{2}, \ldots, e_{k}\right\}$. Suppose that $\operatorname{rank}(H)=m$. The adjacency tensor $\mathcal{A}_{H}$ of $H$ is as follows:

$$
\mathcal{A}_{H}=\left(a_{i_{1}, i_{2}, \ldots, i_{m}}\right), \quad 1 \leq i_{1}, i_{2}, \ldots, i_{m} \leq n .
$$

For all edges $e=\left\{v_{l_{1}}, v_{l_{2}}, \ldots, v_{l_{s}}\right\} \in E$ of cardinality $s \leq m$,

$$
a_{i_{1}, i_{2}, \ldots, i_{m}}=\frac{s}{\alpha(s)}, \quad \text { where } \alpha(s)=\sum_{\substack{k_{1}, \ldots, k_{s} \geq 1 \\ k_{1}+\cdots+k_{s}=m}} \frac{m !}{k_{1} ! k_{2} ! \cdots k_{s} !}
$$

and $i_{1}, i_{2}, \ldots, i_{m}$ are chosen in all possible ways from $L=\left\{l_{1}, \ldots, l_{s}\right\}$ with at least once for each element of the set $L$, while each $k_{i}$ represents the times that $l_{i}$ appears in $\left\{i_{1}, i_{2}, \ldots, i_{m}\right\}$. The other positions of the tensor are zero.

The adjacency tensor is symmetric, while the nonzero elements of adjacency tensor may have different values. Even if the subscript of the elements contains two same indices, the value may be nonzero. Specially, when $H$ is an $m$-uniform hypergraph, we know that the nonzero elements of adjacency tensor are $1 /(m-1)$ !.

Let $H=(V, E)$ be a hypergraph, where $V=\left\{v_{1}, v_{2}, \ldots, v_{n}\right\}$ and $E=\left\{e_{1}, e_{2}, \ldots, e_{k}\right\}$. The degree of a vertex $v_{i}$ is given by

$$
d_{i}=d_{H}\left(v_{i}\right)=\sum_{i_{2}, i_{3}, \ldots, i_{m}=1}^{n} a_{i i_{2} i_{3} \ldots i_{m}}
$$


Let $E_{i}$ be the set of all the edges containing $v_{i}$ and $e=\left\{v_{i}, v_{i_{2}}, \ldots, v_{i_{s}}\right\} \in E_{i}$. We denote an $i$-th component of a vector $\mathcal{A}(e) x$ by

$$
(\mathcal{A}(e) x)_{i}=\frac{s}{\alpha(s)} \sum_{\substack{k_{1} \geq 0, k_{2}, \ldots, k_{s} \geq 1 \\ k_{1}+\cdots+k_{s}=m-1}} \frac{(m-1) !}{k_{1} ! k_{2} ! \cdots k_{s} !} x_{i}^{k_{1}} x_{i_{2}}^{k_{2}} \cdots x_{i_{s}}^{k_{s}},
$$

then we have

$$
(\mathcal{A} x)_{i}=\sum_{e \in E_{i}}(\mathcal{A}(e) x)_{i}
$$

And we also have

$$
x^{T} \mathcal{A}(e) x=\frac{s}{\alpha(s)} \sum_{\substack{k_{1}, k_{2}, \ldots, k_{s} \geq 1 \\ k_{1}+\cdots+k_{s}=m}} \frac{m !}{k_{1} ! k_{2} ! \cdots k_{s} !} x_{i_{1}}^{k_{1}} x_{i_{2}}^{k_{2}} \cdots x_{i_{s}}^{k_{s}},
$$

then we have

$$
x^{T} \mathcal{A} x=\sum_{e \in E} x^{T} \mathcal{A}(e) x
$$

Definition 2.3. [1] Let $H=(V, E)$ be a (general) hypergraph without isolated vertex where $V=\left\{v_{1}, v_{2}, \ldots, v_{n}\right\}$ and $E=\left\{e_{1}, e_{2}, \ldots, e_{k}\right\}$. Let $\operatorname{rank}(H)=m$. We define the Laplacian hypermatrix, $\mathcal{L}_{H}$, of $H=(V, E)$ as $\mathcal{L}_{H}=\mathcal{D}_{H}-\mathcal{A}_{H}=\left(l_{i_{1} i_{2} \ldots i_{m}}\right), 1 \leq$ $i_{1}, i_{2}, \ldots, i_{m} \leq n$, where $\mathcal{D}_{H}=\left(d_{i_{1}, i_{2}, \ldots, i_{m}}\right)$ is the $m$ order $n$ dimensional hypermatrix with $d_{i i \ldots i}=d_{i}$ and others are zero. The signless Laplacian hypermatrix of $G$ is defined as $\mathcal{Q}_{H}=\mathcal{D}_{H}+\mathcal{A}_{H}$.

Let $H=(V, E)$ be a general hypergraph with $\operatorname{rank}(H)=m$. For any edge $e=$ $\left\{v_{i_{1}}, v_{i_{2}}, \ldots, v_{i_{s}}\right\}$, we have

$$
\mathcal{L}(e) x^{m}=\sum_{j=1}^{s} x_{i_{j}}^{m}-\frac{s}{\alpha(s)} x_{m}^{e},
$$

where $x_{m}^{e}=\sum_{\substack{k_{1}, \ldots, k_{s} \geq 1 \\ k_{1}+\cdots+k_{s}=m}} \frac{m !}{k_{1} ! k_{2} ! \cdots k_{s} !} x_{i_{1}}^{k_{1}} \cdots x_{i_{s}}^{k_{s}}$.

And we also have

$$
\mathcal{L} x^{m}=\sum_{e \in E} \mathcal{L}(e) x^{m}
$$

Similarly, we have

$$
\mathcal{Q}(e) x^{m}=\sum_{j=1}^{s} x_{i_{j}}^{m}+\frac{s}{\alpha(s)} x_{m}^{e}
$$

And we also have

$$
\mathcal{Q} x^{m}=\sum_{e \in E} \mathcal{Q}(e) x^{m} .
$$


3. Some properties of signless Laplacian eigenvalues of general hypergraphs In this section, we mainly give some basic properties of signless Laplacian tensors of general hypergraphs.

Theorem 3.1. Let $G$ be a general hypergraph with $n$ vertices and maximum degree $\Delta$ such that $\operatorname{rank}(G)=m$, and let $q$ be an $H$-eigenvalue of $\mathcal{Q}_{G}$. Then we have $|q| \leq 2 \Delta$.

Proof. Let $x=\left(x_{1}, x_{2}, \ldots, x_{n}\right)^{T}$ be an eigenvector of a hypergraph $G$ corresponding to $q$ and $x_{t}=\max \left\{\left|x_{1}\right|,\left|x_{2}\right|, \ldots,\left|x_{n}\right|\right\}$. Without loss of generality, we can assume that $x_{t}=1$.

$$
\begin{aligned}
|q| & =\left|q x_{t}^{m-1}\right|=\left|d_{t} x_{t}^{m-1}+\sum_{i_{2}, i_{3}, \ldots, i_{m}=1}^{n} a_{t i_{2} i_{3} \ldots i_{m}} x_{i_{2}} x_{i_{3}} \cdots x_{i_{m}}\right| \\
& \leq\left|d_{t} x_{t}^{m-1}\right|+\left|\sum_{i_{2}, i_{3}, \ldots, i_{m}=1}^{n} a_{t i_{2} i_{3} \ldots i_{m}} x_{t}^{m-1}\right| \\
& \leq\left|d_{t}\right|\left|x_{t}^{m-1}\right|+\sum_{i_{2}, i_{3}, \ldots, i_{m}=1}^{n}\left|a_{t i_{2} i_{3} \ldots i_{m}}\right|\left|x_{t}\right|^{m-1} \\
& \leq 2 d_{t} \leq 2 \Delta .
\end{aligned}
$$

A hypergraph is called $k$-regular if every vertex has the same degree $k$. By Theorem 3.1 . we have $|q| \leq 2 k$ for a $k$-regular hypergraph.

Corollary 3.2. Let $G$ be a $k$-regular general hypergraph with $\operatorname{rank}(G)=m$ and $n$ vertices. Then $2 k$ is an $H^{++}$-eigenvalue of $\mathcal{Q}_{G}$.

Proof. For a vector $x=(1,1, \ldots, 1)^{T} \in \mathbb{R}^{n}$, and $1 \leq i \leq n$, we have

$$
\left(\mathcal{Q}_{G} x^{m-1}\right)_{i}=d_{i}+\sum_{i_{2}, i_{3}, \ldots, i_{m}=1}^{n} a_{i i_{2} i_{3} \ldots i_{m}}=2 k .
$$

Definition 3.3. [1] Let $\mathcal{A}$ be a nonzero hypermatrix. A pair $(\lambda, x) \in\left(\mathbb{C} \times \mathbb{C}^{n} \backslash\{0\}\right)$ is called an $E$-eigenpair (where $\lambda$ and $x$ are called $E$-eigenvalue and $E$-eigenvector, respectively) if they satisfy the following equations

$$
\mathcal{A} x^{m-1}=\lambda x, \quad \sum_{i=1}^{n} x_{i}^{2}=1 .
$$

We call $(\lambda, x)$ a $Z$-eigenpair if both of them are real.

Theorem 3.4. Let $G$ be a k-regular general hypergraph with $\operatorname{rank}(G)=m$ and $n$ vertices. Then $2 k(1 / \sqrt{n})^{m-2}$ is a $Z$-eigenvalue of $\mathcal{Q}_{G}$. 
Proof. For a vector $x=(1 / \sqrt{n}, 1 / \sqrt{n}, \ldots, 1 / \sqrt{n})^{T} \in \mathbb{R}^{n}$, we have $\sum_{i=1}^{n} x_{i}^{2}=1$. And for $1 \leq i \leq n$, we have

$$
\begin{aligned}
\left(\mathcal{Q}_{G} x^{m-1}\right)_{i} & =d_{i} x^{m-1}+\sum_{i_{2}, i_{3}, \ldots, i_{m}=1}^{n} a_{i i_{2} i_{3} \ldots i_{m}} x_{i_{2}} x_{i_{3}} \cdots x_{i_{m}} \\
& =k\left(\frac{1}{\sqrt{n}}\right)^{m-1}+\left(\frac{1}{\sqrt{n}}\right)^{m-1} \sum_{i_{2}, i_{3}, \ldots, i_{m}=1}^{n} a_{i i_{2} i_{3} \ldots i_{m}} \\
& =2 k\left(\frac{1}{\sqrt{n}}\right)^{m-2} \cdot\left(\frac{1}{\sqrt{n}}\right) .
\end{aligned}
$$

By a similar argument to the proof of Theorem 3.4 in [1], we have the following result.

Theorem 3.5. Let $G$ be a general hypergraph with $n$ vertices and maximum degree $\Delta$ such that $\operatorname{rank}(G)=m$. Let $x=\left(x_{1}, x_{2}, \ldots, x_{n}\right)^{T}$ be a $Z$-eigenvector of $\mathcal{Q}_{G}$ corresponding to an eigenvalue $q$. If $x_{t}=\max \left\{\left|x_{1}\right|,\left|x_{2}\right|, \ldots,\left|x_{n}\right|\right\}$, then we have $|q| \leq 2 \Delta / x_{t}$.

Let $x=\left(x_{1}, x_{2}, \ldots, x_{n}\right)^{T}$ be a vector in $\mathbb{R}^{n}$ and $m \geq s-1$ be an integer. For an edge $e=\left\{v_{l_{1}}, v_{l_{2}}, \ldots, v_{l_{s}}\right\}$ and a vertex $v_{l_{i}}$, we define

$$
x_{m}^{e / v_{l_{i}}}:=\sum x_{i_{1}} x_{i_{2}} \cdots x_{i_{m}}
$$

where the sum is over $i_{1}, i_{2}, \ldots, i_{m}$ chosen in all possible way from $\left\{l_{1}, l_{2}, \ldots, l_{s}\right\}$, such that, all $l_{j}(j \neq i)$ occur at least once.

Definition 3.6. [1] Let $G$ and $H$ be two hypergraphs. The Cartesian product, $G \times H$, of $G$ and $H$ is defined by the vertex set $V(G \times H)=V(G) \times V(H)$ and the edge set $E(G \times H)=\{\{v\} \times e: v \in V(G), e \in E(H)\} \cup\{e \times\{v\}: e \in E(G), v \in V(H)\}$.

The following theorem is similar to the proof of uniform hypergraphs in [4].

Theorem 3.7. Let $G$ and $H$ be two general hypergraphs with $\operatorname{rank}(G)=\operatorname{rank}(H)$. If $q_{G}$ and $q_{H}$ are $H$-eigenvalues for $G$ and $H$, respectively, then $q_{G}+q_{H}$ is an $H$-eigenvalue for $G \times H$.

Proof. Let $n_{1}$ and $n_{2}$ be the number of vertices in $G$ and $H$, respectively. Let $\left(q_{G}, \mathbf{u}\right)$ and $\left(q_{H}, \mathbf{v}\right)$ be $H$-eigenpairs of $\mathcal{Q}_{G}$ and $\mathcal{Q}_{H}$, respectively. Let $\mathbf{w} \in \mathbb{C}^{n_{1} n_{2}}$ be a vector with the entries indexed by the pairs $(a, b) \in\left[n_{1}\right] \times\left[n_{2}\right]$ such that $w(a, b)=u(a) v(b)$. We have

$$
\begin{aligned}
& d_{(a, b)}^{G \times H} w^{m-1}(a, b)+\sum_{e \in E_{(a, b)}} a_{e}^{G \times H} w_{m-1}^{e /(a, b)} \\
= & \left(d_{a}^{G}+d_{b}^{H}\right) w^{m-1}(a, b)+\sum_{e \times\{b\} \in E_{(a, b)}, e \in E_{a}} a_{e}^{G \times H} w_{m-1}^{e \times\{b\} /(a, b)}
\end{aligned}
$$




$$
\begin{aligned}
& +\sum_{\{a\} \times e \in E_{(a, b)}, e \in E_{b}} a_{e}^{G \times H} w_{m-1}^{\{a\} \times e /(a, b)} \\
= & d_{a}^{G} w^{m-1}(a, b)+\sum_{e \in E_{a}(G)} a_{e}^{G \times H} u_{m-1}^{e / a} v^{m-1}(b) \\
& +d_{b}^{H} w^{m-1}(a, b)+\sum_{e \in E_{b}(H)} a_{e}^{G \times H} u^{m-1}(a) v_{m-1}^{e / b} \\
= & d_{a}^{G} u^{m-1}(a) v^{m-1}(b)+v^{m-1}(b) \sum_{e \in E_{a}(G)} a_{e}^{G} u_{m-1}^{e / a} \\
& +d_{b}^{H} u^{m-1}(a) v^{m-1}(b)+u^{m-1}(a) \sum_{e \in E_{b}(H)} a_{e}^{H} v_{m-1}^{e / b} \\
= & v^{m-1}(b) q_{G} u^{m-1}(a)+u^{m-1}(a) q_{H} v^{m-1}(b) \\
= & \left(q_{G}+q_{H}\right) w^{m-1}(a, b),
\end{aligned}
$$

where $d_{(a, b)}^{G \times H}, d_{a}^{G}$ and $d_{b}^{H}$, respectively, are the degree of $(a, b)$ in $G \times H$, the degree of $a$ in $G$ and the degree of $b$ in $H$.

Denote by $\mathbf{e}^{(j)}$ the $j$ th unit vector in $\mathbb{R}^{n}$, i.e., $\mathbf{e}_{i}^{(j)}=1$ if $i=j$ and $\mathbf{e}_{i}^{(j)}=0$ if $i \neq j$, for $i, j=1,2, \ldots, n$.

Theorem 3.8. Let $G$ be a general hypergraph and $s=\min _{e \in E(G)}|e| \geq 2$. For $j=$ $1,2, \ldots, n, d_{j}$ is an $H^{+}$-eigenvalue of $\mathcal{Q}_{G}$ with eigenvector $\mathbf{e}^{(j)}$.

Proof. For a vector $\mathbf{e}^{(j)}$, we can easily verify that for $j=1,2, \ldots, n, d_{j}$ is an $H^{+}$-eigenvalue of $\mathcal{Q}_{G}$ with eigenvector $\mathbf{e}^{(j)}$ by the characteristic equation of the signless Laplacian tensor.

Theorem 3.9. Let $G$ be a general hypergraph with $\operatorname{rank}(G)=m$ and $n$ vertices. Then we have

$$
\max \{\Delta, 2 \bar{d}\} \leq q_{1} \leq 2 \Delta
$$

where $\bar{d}=\frac{1}{n} \sum_{i=1}^{n} d_{i}$

Proof. By the characteristic equation of the signless Laplacian tensor, it is obvious that $\Delta \leq q_{1} \leq 2 \Delta$. Let $x=\left(\frac{1}{n^{1 / m}}, \frac{1}{n^{1 / m}}, \ldots, \frac{1}{n^{1 / m}}\right)^{T}$, we have

$$
q_{1} \geq \mathcal{Q}_{G} x^{m}=\sum_{i=1}^{n} d_{i} x_{i}^{m}+\sum_{i=1}^{n} \sum_{i_{2}, i_{3}, \ldots, i_{m}=1}^{n} a_{i i_{2} i_{3} \ldots i_{m}} x_{i} x_{i_{2}} x_{i_{3}} \cdots x_{i_{m}}=2 \frac{1}{n} \sum_{i=1}^{n} d_{i} .
$$

Thus,

$$
\max \{\Delta, 2 \bar{d}\} \leq q_{1} \leq 2 \Delta
$$

By a similar argument to the proof of Theorem 15 of [20], we have the following result. 
Theorem 3.10. The smallest signless Laplacian $H^{+}$-eigenvalue of $G$ is $\delta$. We always have

$$
\delta=\min \left\{\mathcal{Q} x^{k}: x \in \mathbb{R}_{+}^{n}, \sum_{i=1}^{n} x_{i}^{k}=1\right\} .
$$

4. Edge connectivity and $H^{+}$-eigenvalues of sub-tensors of the normalized Laplacian tensors

Let $S$ be a proper nonempty subset of $V$, and $\bar{S}=V \backslash S$ be the complement of $S$ in $G$. The edge set $E$ is partitioned into three parts $E(S), E(\bar{S})$ and $E(S, \bar{S})$. The edge set $E(S)$ (resp. $E(\bar{S})$ ) consists of edges whose vertices are all in $S$ (resp. $\bar{S}$ ). The edge set $E(S, \bar{S})$ consists of edges whose vertices are in both $S$ and $\bar{S}$. We call $E(S, \bar{S})$ an edge cut of $G$. If we delete all the edges of $E(S, \bar{S})$ from $G$, then $G$ is separated into two hypergraphs $G[S]=(S, E(S))$ and $G[\bar{S}]=(\bar{S}, E(\bar{S})$ ) (note that the ranks of $G[S]$ and $G[\bar{S}]$ may be different). For an edge $e_{p} \in E(S, \bar{S}), t\left(e_{p}\right)$ is the number of vertices contained in $S$ of $e_{p}$. For all edges $e_{p} \in E(S, \bar{S})$, the average value of such $t\left(e_{p}\right)$ is denoted $t(S)$. We call the minimum (resp. maximum) cardinality of such an edge cut the edge connectivity (resp. maximum cut) of $G$, and denoted by $e(G)$ (resp. $c(G)$ ).

For each edge $e=\left\{v_{i_{1}}, v_{i_{2}}, \ldots, v_{i_{t}}\right\}$, denote by $S_{e}$ the collection of all the ordered multisets extended from $e$, that is $\left|S_{e}\right|=\alpha(t)$, and $S_{E(G)}=\bigcup_{e \in E(G)} S_{e}$ represent all the possible ordered multisets extended from the edges in $E(G)$.

In the following, let $G$ be a hypergraph with $n$ vertices and $k$ edges. When the hypergraph $G$ is uniform, Theorem 4.1 is exactly Proposition 16 of Qi 20].

Theorem 4.1. Let $G$ be a general hypergraph with $n$ vertices, average degree $\bar{d}$, minimum degree $\delta$ and $\operatorname{rank}(G)=m$. Let $m \geq s=\min _{e \in E(G)}|e| \geq 2$.

(i) If $m-2 s<0$, then, for any nonempty proper subset $S$ of $V(G)$, we have

$$
e(G) \leq|E(S, \bar{S})| \leq \frac{(2 \bar{d}-\delta) n}{2 s-m} .
$$

(ii) If $n \leq 2 s-1$, then $e(G)=\delta$.

Proof. (i) Let $S$ be a nonempty proper subset of $V$. Let $x=\frac{1}{|S|^{1 / m}} \sum_{i \in S} \mathbf{e}^{(i)}$, where $\mathbf{e}^{(i)}$ is a vector whose $i$-th element is 1 , and other elements are 0 . For $e_{p} \in E(S)$, by Definition 2.3, we have

$$
\begin{aligned}
\mathcal{Q}_{G}\left(e_{p}\right) x^{m} & =\left|e_{p}\right| \cdot \frac{1}{|S|}+\frac{\left|e_{p}\right|}{\alpha\left(\left|e_{p}\right|\right)} \sum_{e_{p}^{\prime} \in S_{e_{p}}} x^{e_{p}^{\prime}}=\left|e_{p}\right| \cdot \frac{1}{|S|}+\frac{\left|e_{p}\right|}{\alpha\left(\left|e_{p}\right|\right)} \sum_{e_{p}^{\prime} \in S_{e_{p}}} \frac{1}{|S|} \\
& =\left|e_{p}\right| \cdot \frac{1}{|S|}+\frac{\left|e_{p}\right|}{\alpha\left(\left|e_{p}\right|\right)} \cdot \alpha\left(\left|e_{p}\right|\right) \cdot \frac{1}{|S|}=\frac{2\left|e_{p}\right|}{|S|} .
\end{aligned}
$$


For $e_{p} \in E(\bar{S})$, it is obvious that

$$
\mathcal{Q}_{G}\left(e_{p}\right) x^{m}=0 \text {. }
$$

For $e_{p} \in E(S, \bar{S})$, it is obvious that

$$
\mathcal{Q}_{G}\left(e_{p}\right) x^{m}=\frac{t\left(e_{p}\right)}{|S|} .
$$

Thus,

$$
\begin{aligned}
\mathcal{Q}_{G} x^{m} & =\left(\sum_{e_{p} \in E(S)}+\sum_{e_{p} \in E(\bar{S})}+\sum_{e_{p} \in E(S, \bar{S})}\right) \mathcal{Q}_{G}\left(e_{p}\right) x^{m} \\
& =\sum_{e_{p} \in E(S)} \frac{2\left|e_{p}\right|}{|S|}+\sum_{e_{p} \in E(S, \bar{S})} \frac{t\left(e_{p}\right)}{|S|}=\sum_{e_{p} \in E(S)} \frac{2\left|e_{p}\right|}{|S|}+\frac{t(S)}{|S|}|E(S, \bar{S})| .
\end{aligned}
$$

Moreover, by Theorem 3.10, we have

$$
\delta|S| \leq \sum_{e_{p} \in E(S)} 2\left|e_{p}\right|+t(S)|E(S, \bar{S})| .
$$

Similarly, letting $y=\frac{1}{|\bar{S}|^{1 / m}} \sum_{i \in \bar{S}} \mathbf{e}^{(i)}$, we have

$$
\mathcal{Q}_{G} x^{m}=\left(\sum_{e_{p} \in E(S)}+\sum_{e_{p} \in E(\bar{S})}+\sum_{e_{p} \in E(S, \bar{S})}\right) \mathcal{Q}_{G}\left(e_{p}\right) x^{m}=\sum_{e_{p} \in E(\bar{S})} \frac{2\left|e_{p}\right|}{|\bar{S}|}+\frac{t(\bar{S})}{|\bar{S}|}|E(S, \bar{S})| .
$$

By Theorem 3.10, we have

$$
\delta|\bar{S}| \leq \sum_{e_{p} \in E(\bar{S})} 2\left|e_{p}\right|+t(\bar{S})|E(S, \bar{S})| .
$$

By Inequalities 4.1) and (4.2), we have

$$
\begin{aligned}
\delta n & \leq \sum_{e_{p} \in E(S)} 2\left|e_{p}\right|+t(S)|E(S, \bar{S})|+\sum_{e_{p} \in E(\bar{S})} 2\left|e_{p}\right|+t(\bar{S})|E(S, \bar{S})| \\
& =\sum_{e_{p} \in E(G)} 2\left|e_{p}\right|-\sum_{e_{p} \in E(S, \bar{S})} 2\left|e_{p}\right|+(t(S)+t(\bar{S}))|E(S, \bar{S})| \\
& \leq 2 \bar{d} n-2 s|E(S, \bar{S})|+m|E(S, \bar{S})| \\
& =2 \bar{d} n-(2 s-m)|E(S, \bar{S})| .
\end{aligned}
$$

If $m-2 s<0$, then we have

$$
e(G) \leq|E(S, \bar{S})| \leq \frac{(2 \bar{d}-\delta) n}{2 s-m}
$$


(ii) If $n \leq 2 s-1$, then either $|S|<s$ or $|\bar{S}|<s$. Without loss of generality, we may assume that $|S|<s$. Then $E(S)=\emptyset$, that is, $|E(S)|=0$. Thus, by 4.1), we have

$$
|S| \delta \leq t(S)|E(S, \bar{S})|
$$

Since $t(S) \leq|S|$, we have

$$
\delta \leq|E(S, \bar{S})|
$$

Thus, $e(G) \geq \delta$.

Assume that $d_{j}=\delta$. Let $S=\{j\}$, then

$$
|E(S, \bar{S})|=d_{j}=\delta
$$

Thus, $e(G)=\delta$.

Let $G=(V, E)$ be a general hypergraph with $\operatorname{rank}(G)=m$. Similar to Qi $[20$ the analytic connectivity $\alpha(G)$ of $G$ is defined as

$$
\alpha(G)=\min _{j=1,2, \ldots, n}\left\{\min \left\{\mathcal{L} x^{m} \mid x \in \mathbb{R}_{+}^{n}, \sum_{i=1}^{n} x_{i}^{m}=1, x_{j}=0\right\}\right\} .
$$

When the hypergraph $G$ is uniform, Theorem 4.2 is exactly Proposition 18 of Qi [20].

Theorem 4.2. Let $G$ be a general hypergraph with $n$ vertices and $\operatorname{rank}(G)=m$. Then we have

$$
e(G) \geq \frac{n}{m} \alpha(G)
$$

Proof. Let $s=\min _{e \in E(G)}|e|$, and let $S$ be a nonempty proper subset of $V$. Then there is a $j \notin S$ such that

$$
\min \left\{\mathcal{L}_{G} x^{m}: x \in \mathbb{R}_{+}^{n}, \sum_{i=1}^{n} x_{i}^{m}=1, x_{j}=0\right\} \geq \alpha(G)
$$

Let $x=\frac{1}{|S|^{1 / m}} \sum_{i \in S} \mathbf{e}^{(i)}$. It is clear that $x$ is a feasible point of the minimization problem in 4.3 . For $e_{p} \in E(S)$, by Definition 2.3, we have

$$
\mathcal{L}_{G}\left(e_{p}\right) x^{m}=\left|e_{p}\right| \cdot \frac{1}{|S|}-\frac{\left|e_{p}\right|}{\alpha\left(\left|e_{p}\right|\right)} \cdot \alpha\left(\left|e_{p}\right|\right) \cdot \frac{1}{|S|}=0 .
$$

For $e_{p} \in E(\bar{S})$, it is obvious that

$$
\mathcal{L}_{G}\left(e_{p}\right) x^{m}=0
$$

For $e_{p} \in E(S, \bar{S})$, it is obvious that

$$
\mathcal{L}_{G}\left(e_{p}\right) x^{m}=\frac{t\left(e_{p}\right)}{|S|} .
$$


Thus,

$$
\begin{aligned}
\mathcal{L}_{G} x^{m} & =\left(\sum_{e_{p} \in E(S)}+\sum_{e_{p} \in E(\bar{S})}+\sum_{e_{p} \in E(S, \bar{S})}\right) \mathcal{L}_{G}\left(e_{p}\right) x^{m} \\
& =\sum_{e_{p} \in E(S, \bar{S})} \frac{t\left(e_{p}\right)}{|S|}=\frac{t(S)}{|S|}|E(S, \bar{S})| .
\end{aligned}
$$

By Inequality (4.3), we have

$$
\alpha(G)|S| \leq t(S)|E(S, \bar{S})|
$$

Similarly, letting $y=\frac{1}{|\bar{S}|^{1 / m}} \sum_{i \in \bar{S}} \mathbf{e}^{(i)}$, we have

$$
\mathcal{L}_{G} x^{m}=\left(\sum_{e_{p} \in E(S)}+\sum_{e_{p} \in E(\bar{S})}+\sum_{e_{p} \in E(S, \bar{S})}\right) \mathcal{L}_{G}\left(e_{p}\right) x^{m}=\frac{t(\bar{S})}{|\bar{S}|}|E(S, \bar{S})|
$$

and

$$
\alpha(G)|\bar{S}| \leq t(\bar{S})|E(S, \bar{S})|
$$

Thus, by Inequalities (4.4) and 4.5), we have

$$
\alpha(G) n \leq t(S)|E(S, \bar{S})|+t(\bar{S})|E(S, \bar{S})| \leq m|E(S, \bar{S})|
$$

Then, we have

$$
|E(S, \bar{S})| \geq \frac{n}{m} \alpha(G)
$$

Hence,

$$
e(G) \geq \frac{n}{m} \alpha(G)
$$

By Theorem 4.2 and $e(G) \leq \delta$, we note $\alpha(G) \leq \frac{m}{n} \delta$ for a hypergraph with rank $=m$ and minimum degree $\delta$.

Motivated by the idea of $\mathrm{Li}$ et al. [11], we have the following theorem. When $G$ is an $m$-uniform hypergraph, the upper bound in Theorem 4.3 is $\min \left\{\frac{d_{i_{1}}+d_{i_{2}}+\cdots+d_{i_{m}}-m}{m} \mid\right.$ $\left.\left\{v_{i_{1}}, v_{i_{2}}, \ldots, v_{i_{m}}\right\} \in E(G)\right\}$, which is exactly the upper bound of Theorem 3.9 in Li et al. [11].

Theorem 4.3. Let $G$ be a general hypergraph with $\operatorname{rank}(G)=m, n$ vertices and more than one edge. Then we have

$$
\alpha(G) \leq \min \left\{\frac{d_{i_{1}}+d_{i_{2}}+\cdots+d_{i_{l}}-l}{l} \mid\left\{v_{i_{1}}, v_{i_{2}}, \ldots, v_{i_{l}}\right\} \in E(G)\right\} .
$$


Proof. Let $e=\left\{v_{i_{1}}, v_{i_{2}}, \ldots, v_{i_{l}}\right\} \in E(G)$ be an edge of $G$ and let $x=\frac{1}{l^{1 / m}} \sum_{i \in e} \mathbf{e}^{(i)}$ be a vector. It is obvious $\sum_{i=1}^{n} x_{i}^{m}=1$. Thus, we have

$$
\begin{aligned}
\alpha(G) & \leq \mathcal{L}_{G} x^{m}=\sum_{e_{p} \in E(G)} \mathcal{L}_{G}\left(e_{p}\right) x^{m} \\
& =d_{i_{1}} x_{i_{1}}^{m}+d_{i_{2}} x_{i_{2}}^{m}+\cdots+d_{i_{l}} x_{i_{l}}^{m}-\sum_{e^{\prime} \in S_{e}} \frac{l}{\alpha(l)} \frac{1}{l} \\
& =d_{i_{1}} \frac{1}{l}+d_{i_{2}} \frac{1}{l}+\cdots+d_{i_{l}} \frac{1}{l}-1=\frac{d_{i_{1}}+d_{i_{2}}+\cdots+d_{i_{l}}-l}{l} .
\end{aligned}
$$

Let $G$ be a general hypergraph with $n$ vertices and $k$ edges, and $\operatorname{rank}(G)=m$. The normalized adjacency tensor $\mathfrak{A}$, which is an $m$-th order $n$-dimensional symmetric nonnegative tensor, is defined as: for any edge $e=\left\{v_{l_{1}}, v_{l_{2}}, \ldots, v_{l_{s}}\right\} \in E$ of cardinality $s \leq m$,

$$
a_{i_{1}, i_{2}, \ldots, i_{m}}=\frac{s}{\alpha(s)} \prod_{j=1}^{m} \frac{1}{\sqrt[m]{d\left(v_{i_{j}}\right)}}, \quad \text { where } \alpha(s)=\sum_{\substack{k_{1}, \ldots, k_{s} \geq 1 \\ k_{1}+\cdots+k_{s}=m}} \frac{m !}{k_{1} ! k_{2} ! \cdots k_{s} !},
$$

and $i_{1}, i_{2}, \ldots, i_{m}$ are chosen in all possible ways from $\left\{l_{1}, l_{2}, \ldots, l_{s}\right\}$ with at least once for each element of the set. The rest of the positions are zero.

The normalized Laplacian tensor $\mathfrak{L}$, which is an $m$-th order $n$-dimensional symmetric tensor, is defined as

$$
\mathfrak{L}=\mathfrak{I}-\mathfrak{A},
$$

where $\mathfrak{I}$ is an $m$-th order $n$-dimensional diagonal tensor with the $i$-th diagonal element $j_{i i \ldots i}=1$, and zero otherwise.

In the following, we gave some results of sub-tensors of the normalized Laplacian tensors.

Definition 4.4. [8] Let $\mathcal{T}$ be a real tensor with order $k$ dimensional $n$ and $s \in[n]$. The $k$ th order $s$-dimensional tensor $\mathcal{U}$ with entries $u_{i_{1}, i_{2}, \ldots, i_{k}}=t_{i_{1}, i_{2}, \ldots, i_{k}}$ for all $i_{1}, i_{2}, \ldots, i_{k} \in[s]$ is called the sub-tensor of $\mathcal{T}$ associated to the subset $S:=\left\{j_{1}, j_{2}, \ldots, j_{s}\right\}$. We usually denoted $\mathcal{U}$ as $\mathcal{T}_{S}$.

Let $S \subseteq[n]$ be nonempty and let $\kappa(S)$ be the smallest $H^{+}$-eigenvalue of normalized Laplacian sub-tensor $\mathfrak{L}_{S}$. The proof of the following theorem is similar to that of uniform hypergraphs in [8]. Hence, we omit its proof.

Theorem 4.5. Let $G$ be a general hypergraph on $n$ vertices without isolated vertices and $\operatorname{rank}(G)=m$. Let $S \subseteq[n]$ be nonempty. Then we have $\kappa(S)=1-\rho\left(\mathfrak{A}_{S}\right)$, and

$$
\kappa(S)=\min \left\{\mathfrak{L} y^{m} \mid y \in \mathbb{R}_{+}^{n}, \sum_{i \in[n]} y_{i}^{m}=1, y_{i}=0, \forall i \in \bar{S}\right\},
$$

where $\rho\left(\mathfrak{A}_{S}\right)$ is the spectral radius of normalized adjacency tensor $\mathfrak{A}_{S}$. 
Corollary 4.6. Let $G$ be a general hypergraph on $n$ vertices without isolated vertices and $\operatorname{rank}(G)=m$. Let $S, T \subseteq[n]$ be nonempty such that $S \subset T$. Then we have $\kappa(T) \leq \kappa(S)$.

Proof. By Theorem 4.5, it is obvious that Corollary 4.6 holds.

Let $\delta=\min _{i \in[n]} d_{i}$ and $\Delta=\max _{i \in[n]} d_{i}$. For a nonempty subset $S \subset V$, define $\operatorname{vol}(S)=\sum_{i \in S} d_{i}$ as the volume of $S$. Let $\operatorname{vol}(\emptyset)=0$. The volume $\operatorname{vol}([n])$ of a hypergraph is simply denoted as $d_{\mathrm{vol}}$. For a subset $S \subset[n]=\{1,2, \ldots, n\}$, we use $E_{S}$ to denote the set of edges $\{e \in E \mid S \cap e \neq \emptyset\}$.

Theorem 4.7. Let $G$ be a general hypergraph on $n$ vertices without isolated vertices and $\operatorname{rank}(G)=m$, and $s=\min _{e \in E(G)}|e| \geq 2$. For any nonempty $S \subseteq[n]$, we have

$$
\kappa(S) \leq \frac{m(s-1) \operatorname{vol}(\bar{S})+(m-s) d_{\mathrm{vol}}-s(m-s)}{m \operatorname{vol}(S)} .
$$

In particular, for any $i \in[n]$, we have

$$
\kappa(\overline{\{i\}}) \leq \frac{m(s-1) \Delta+(m-s) d_{\mathrm{vol}}-s(m-s)}{m\left(d_{\mathrm{vol}}-\Delta\right)} .
$$

Proof. If $S=V$, then it is obvious that $\kappa(S)=\kappa(V)=0$ and the result holds. Thus we may assume that $S \neq V$. Let $\mathbf{y}=\left(y_{1}, y_{2}, \ldots, y_{n}\right)^{T}$ be the vector with its $j$-th element being $\sqrt[m]{d_{j}} / \sqrt[m]{\operatorname{vol}(S)}$ for all $j \in S$ and $y_{j}=0$ for $j \in \bar{S}$. Then, by Theorem 4.5, we have that

$$
\begin{aligned}
\kappa(S) & \leq \mathfrak{L y}^{m}=1-\mathfrak{A}_{S} \mathbf{y}^{m}=1-\sum_{e \in E \backslash E_{\bar{S}}} \mathfrak{A}_{S}(e) \mathbf{y}^{m} \\
& =1-\sum_{e \in E \backslash E_{\bar{S}}, e \mid=l} \frac{l}{\alpha(l)} \sum_{\substack{k_{1}, k_{2}, \ldots, k_{l} \geq 1 \\
k_{1}+k_{2}+\cdots+k_{l}=m}} \frac{m !}{k_{1} ! \cdots k_{l} !} \frac{y_{i_{1}}^{k_{1}} y_{i_{2}}^{k_{2}} \cdots y_{i_{l}}^{k_{l}}}{d_{i_{1}}^{k_{1} / m} d_{i_{2}}^{k_{2} / m} \cdots d_{i_{l}}^{k_{l} / m}} \\
& \leq 1-s \sum_{e \in E \backslash E_{\bar{S}}} \frac{1}{\operatorname{vol}(S)}=1-s \frac{|E|-\left|E_{\bar{S}}\right|}{\operatorname{vol}(S)} \\
& \leq \frac{\operatorname{vol}(S)+s\left|E_{\bar{S}}\right|-\frac{s d_{\mathrm{vol}}+s(m-s)}{m}}{\operatorname{vol}(S)} \leq \frac{\operatorname{vol}(S)+s \operatorname{vol}(\bar{S})-\frac{s d_{\mathrm{vol}}+s(m-s)}{m}}{\operatorname{vol}(S)} \\
& =\frac{m(s-1) \operatorname{vol}(\bar{S})+(m-s) d_{\mathrm{vol}}-s(m-s)}{m \operatorname{vol}(S)} .
\end{aligned}
$$

Since $d_{\mathrm{vol}} \leq s+m(|E|-1)$, that is, $|E| \geq\left(d_{\mathrm{vol}}+m-s\right) / m$, we know that the inequality above is true.

Moreover, for any $i \in[n]$, it is obvious that

$$
\kappa(\overline{\{i\}}) \leq \frac{m(s-1) \Delta+(m-s) d_{\mathrm{vol}}-s(m-s)}{m\left(d_{\mathrm{vol}}-\Delta\right)} .
$$


Example 4.8. Let $G=(V, E)$ be a general hypergraph, where $V=\{1,2,3,4,5\}$ and $E=\{\{1,2,3,4\},\{2,3,4\},\{3,4,5\},\{1,5\}\}$. We know that $m(=\operatorname{rank}(G))=4$ and $s=2$. Let $S=\{1,2,3,4\}$, then we have $\operatorname{vol}(S)=d_{1}+d_{2}+d_{3}+d_{4}=2+2+3+3=10$, $\operatorname{vol}(\bar{S})=d_{5}=2$, and $d_{\mathrm{vol}}=\operatorname{vol}(S)+\operatorname{vol}(\bar{S})=12$. By Theorem 4.7, we have that

$$
\kappa(S) \leq \frac{4(2-1) \operatorname{vol}(\bar{S})+(4-2) d_{\mathrm{vol}}-2(4-2)}{4 \operatorname{vol}(S)}=\frac{8+24-4}{40}=\frac{7}{10}
$$

Theorem 4.9. Let $G$ be a general hypergraph on $n$ vertices without isolated vertices and $\operatorname{rank}(G)=m$, and $s=\min _{e \in E(G)}|e| \geq 2$. Then we have

$$
\min \kappa(\overline{\{i\}}) \leq \frac{d_{\mathrm{vol}}+s(e(G)-|E|)}{d_{\mathrm{vol}}}
$$

Proof. Let $S \subseteq[n]$ be nonempty. Let $\mathbf{y}=\left(y_{1}, y_{2}, \ldots, y_{n}\right)^{T}$ be the vector with its $j$-th element being $\sqrt[m]{d_{j}} / \sqrt[m]{\operatorname{vol}(S)}$ for all $i \in S$ and $y_{j}=0$ for $j \in \bar{S}$. Then, by Theorem 4.5 . we have that

$$
\begin{aligned}
\kappa(S) & \leq \mathfrak{L} y^{m}=1-\sum_{e \in E(S),|e|=l} \frac{l}{\alpha(l)} \sum_{\begin{array}{c}
k_{1}, k_{2}, \ldots, k_{l} \geq 1 \\
k_{1}+k_{2}+\cdots+k_{l}=m
\end{array}} \frac{m !}{k_{1} ! \cdots k_{l} !} \frac{y_{i_{1}}^{k_{1}} y_{i_{2}}^{k_{2}} \cdots y_{i_{l}}^{k_{l}}}{d_{i_{1}}^{k_{1} / m} d_{i_{2}}^{k_{2} / m} \cdots d_{i_{l}}^{k_{l} / m}} \\
& \leq 1-s \sum_{e \in E(S)} \frac{1}{\operatorname{vol}(S)}=1-s \frac{|E(S)|}{\operatorname{vol}(S)} .
\end{aligned}
$$

Similarly, we have that

$$
\kappa(\bar{S}) \leq 1-s \frac{|E(\bar{S})|}{\operatorname{vol}(\bar{S})} .
$$

Hence,

$$
\begin{aligned}
\kappa(S) \operatorname{vol}(S)+\kappa(\bar{S}) \operatorname{vol}(\bar{S}) & \leq \operatorname{vol}(S)+\operatorname{vol}(\bar{S})-s(|E(S)|+|E(\bar{S})|) \\
& =d_{\mathrm{vol}}-s(|E|-|E(S, \bar{S})|)
\end{aligned}
$$

It is obvious that $S \subseteq \overline{\{r\}}$ and $\bar{S} \subseteq \overline{\{t\}}$ for some $r$ and $t$ respectively. Without loss of generality, we assume that $\kappa(S) \leq \kappa(\bar{S})$. By Corollary 4.6. we have that

$$
\begin{aligned}
d_{\mathrm{vol}} \min _{i \in[n]} \kappa(\overline{\{i\}}) & \leq d_{\mathrm{vol}} \min \{\kappa(\overline{\{r\}}), \kappa(\overline{\{t\}})\} \leq d_{\mathrm{vol}} \kappa(S)=(\operatorname{vol}(S)+\operatorname{vol}(\bar{S})) \kappa(S) \\
& \leq \operatorname{vol}(S) \kappa(S)+\operatorname{vol}(\bar{S}) \kappa(\bar{S}) \leq d_{\mathrm{vol}}-s(|E|-|E(S, \bar{S})|) .
\end{aligned}
$$

Thus,

$$
\min \kappa(\overline{\{i\}}) \leq \frac{d_{\mathrm{vol}}+s(e(G)-|E|)}{d_{\mathrm{vol}}}
$$




\section{Acknowledgments}

The authors would like to thank an anonymous referee for his or her valuable comments and suggestions to improve the presentation of this paper. This work is supported by the National Natural Science Foundation of China (Grant No. 11871398), the Natural Science Basic Research Plan in Shaanxi Province of China (Program No. 2018JM1032), the Fundamental Research Funds for the Central Universities (No. 3102019ghjd003) and the Seed Foundation of Innovation and Creation for Graduate Students in Northwestern Polytechnical University (Grant No. ZZ2019031).

\section{References}

[1] A. Banerjee, A. Char and B. Mondal, Spectra of general hypergraphs, Linear Algebra Appl. 518 (2017), 14-30.

[2] A. E. Brouwer and W. H. Haemers, Spectra of Graphs, Universitext, Springer, New York, 2012.

[3] S. M. Cioabă, D. A. Gregory and V. Nikiforov, Extreme eigenvalues of nonregular graphs, J. Combin. Theory Ser. B 97 (2007), no. 3, 483-486.

[4] J. Cooper and A. Dutle, Spectra of uniform hypergraphs, Linear Algebra Appl. 436 (2012), no. 9, 3268-3292.

[5] D. Cvetković, P. Rowlinson and S. Simić, An Introduction to the Theory of Graph Spectra, London Mathematical Society Student Texts 75, Cambridge University Press, Cambridge, 2010.

[6] C. Duan, L. Wang and P. Xiao, The largest signless Laplacian spectral radius of uniform supertrees with diameter and pendent edges (vertices), arXiv:1807.05955.

[7] C. Duan, L. Wang, P. Xiao and X. Li, The (signless Laplacian) spectral radius (of subgraphs) of uniform hypergraphs, submitted, 2017.

[8] S. Hu and L. Qi, The Laplacian of a uniform hypergraph, J. Comb. Optim. 29 (2015), no. $2,331-366$.

[9] H. Li, J.-Y. Shao and L. Qi, The extremal spectral radii of k-uniform supertrees, J. Comb. Optim. 32 (2016), no. 3, 741-764.

[10] H. Li, J. Zhou and C. Bu, Principal eigenvectors and spectral radii of uniform hypergraphs, Linear Algebra Appl. 544 (2018), 273-285. 
[11] W. Li, J. Cooper and A. Chang, Analytic connectivity of k-uniform hypergraphs, Linear Multilinear Algebra 65 (2017), no. 6, 1247-1259.

[12] L.-H. Lim, Singular values and eigenvalues of tensors: A variational approach, Proceedings of the 1st IEEE international Workshop on Computational Advances in Multi-sensor Adaptive Processing, (2005), 129-132.

[13] Eigenvalues of tensors and some very basic spectral hypergraph theory, Matrix Computations and Scientific Computing Seminar, April 16, 2008. http://www.stat.uchicago.edu/lekheng/work/mcsc2.

[14] H. Lin, B. Mo, B. Zhou and W. Weng, Sharp bounds for ordinary and signless Laplacian spectral radii of uniform hypergraphs, Appl. Math. Comput. 285 (2016), 217-227.

[15] L. Liu, L. Kang and X. Yuan, On the principal eigenvectors of uniform hypergraphs, Linear Algebra Appl. 511 (2016), 430-446.

[16] L. Lu and S. Man, Connected hypergraphs with small spectral radius, Linear Algebra Appl. 509 (2016), 206-227.

[17] C. Ouyang, L. Qi and X. Yuan, The first few unicyclic and bicyclic hypergraphs with largest spectral radii, Linear Algebra Appl. 527 (2017), 141-162.

[18] L. Qi, Eigenvalues of a real supersymmetric tensor, J. Symbolic Comput. 40 (2005), no. $6,1302-1324$.

[19] Symmetric nonnegative tensors and copositive tensors, Linear Algebra Appl. 439 (2013), no. 1, 228-238.

[20],$H^{+}$-eigenvalues of Laplacian and signless Laplacian tensors, Commun. Math. Sci. 12 (2014), no. 6, 1045-1064.

[21] P. Xiao and L. Wang, The maximum spectral radius of uniform hypergraphs with given number of pendant edges, Linear Multilinear Algebra 67 (2019), no. 7, 1392-1403.

[22] P. Xiao, L. Wang and Y. Du, The first two largest spectral radii of uniform supertrees with given diameter, Linear Algebra Appl. 536 (2018), 103-119.

[23] P. Xiao, L. Wang and Y. Lu, The maximum spectral radii of uniform supertrees with given degree sequences, Linear Algebra Appl. 523 (2017), 33-45.

[24] X. Yuan, J. Shao and H. Shan, Ordering of some uniform supertrees with larger spectral radii, Linear Algebra Appl. 495 (2016), 206-222. 
[25] J.-J. Yue, L.-P. Zhang, M. Lu and L.-Q. Qi, The adjacency and signless Laplacian spectra of cored hypergraphs and power hypergraphs, J. Oper. Res. Soc. China 5 (2017), no. $1,27-43$.

[26] W. Zhang, L. Liu, L. Kang and Y. Bai, Some properties of the spectral radius for general hypergraphs, Linear Algebra Appl. 513 (2017), 103-119.

Cunxiang Duan, Ligong Wang and Xihe Li

Department of Applied Mathematics, School of Science, Northwestern Polytechnical

University, Xi'an, Shaanxi 710072, China

and

Xi'an-Budapest Joint Research Center for Combinatorics, Northwestern Polytechnical

University, Xi'an, Shaanxi 710129, China

E-mail address: cxduanmath@163.com, lgwangmath@163.com, 1xhdhr@163.com 